\title{
Investigation of Eigen Frequencies and Eigen Modes of Rooms Due To Low Frequency Noise
}

\author{
A.M.Shehap \\ Professor of Physics \\ Department of Physics, Faculty \\ of Science, Cairo University, \\ Giza,Egypt
}

\author{
Hany A.Shawky \\ Department of Acoustics, Mass \\ and force metrology division \\ National institute for \\ standards(NIS) \\ Tersa st.,Giza,Egypt
}

\author{
Tarek M.El-Basheer \\ Department of Acoustics, Mass \\ and force metrology division \\ National institute for \\ standards(NIS) \\ Tersa st.,Giza,Egypt
}

\begin{abstract}
There are rapid social, economic and technological changes, and the numbers of industrial noise sources are growing higher which are affecting a large number of individuals in past decades. Low frequency noise (LFN), the frequency of which ranges below $250 \mathrm{~Hz}$, is generated by many machines commonly used in the working environment, such as fans, ventilation systems, heat pumps, diesel engines, blowers, compressors, and so on. Sound pressure levels (SPLs) at low frequencies (20 to $200 \mathrm{~Hz}$ ) are strongly dependent on measurement position in normal-sized rooms. The variation of SPL can be above $20 \mathrm{~dB}$ between different measurement locations. Resonance frequency, called modes are present in every room. For low frequency modes we have to investigate the resonance behavior of room as a whole. This paper demonstrates the use of COMSOL multiphysics to predict the acoustic response of a room such as laboratory in Shopra Elkheima electrical power plant.
\end{abstract}

Keywords: low frequency noise(LFN), electrical power plant, resonance frequency, eigenmodes, eigenfrequencies.

\section{INTRODUCTION}

The generation of the standing waves due to LFN is a commonly confronted problem in everyday life. The increment of noise can be caused by standing waves, if some requirements are fulfilled. Usually at these conditions the people complaints about insomnia, migraines, lack of concentration, or other, even more serious, health problems.[1-3]. The sound fields in rooms at low frequencies are complicated because of individual room modes. The SPL measurements become very uncertain because measurement in one position, or even a few positions, cannot be used to describe the experienced sound field in general. Some measurement methods have been specially designed to reduce the uncertainty and to improve the reproducibility at low frequencies, but still it is not clear worldwide which method are the most appropriate for practical use. Some methods focus on the experienced sound field. That is, they suggest measurements only on occupant's positions in the room. The repeatability can be low because different measurement technicians may have different criteria to choose the measurement locations. Other methods perform the measurements close to the corners where the maximum SPL is normally found. The repeatability of those methods is better but it is not clear how the measured values should be compared against the target values.

Uneven distribution of sound leads to uncertainties in noise level measurements in rooms especially at low frequencies. All standardized room acoustic measurement methods, e.g., ISO 10052, ISO 140 series, ISO 354, and ISO 374x series, presume that the acoustic field of the room is diffuse. Ideally, the diffuse sound field presupposes that the sound enters to any point of the room evenly from all directions, that is, the sound intensity vector is zero.

\section{ROOM MODES}

Room modes, sometimes called standing waves, result from a coincidence where the sound wave, examined in a certain point, returns to the same point after two or more reflections from the room boundaries and interferes with the original sound wave in the same phase. The sound travels the same path ideally infinite times. As a result of this, the sound pressure level depends strongly on location. Room modes appear at certain frequencies, called room resonance frequencies or eigenfrequencies where the length of the path described above is a multiple of wavelength.

At room modes there will be fixed points in the room where the waves will amplify each other to produce antinodes (sound pressure is high) and other points where complete or partial cancellation occurs to produce a node (sound pressure is zero). These points determine the highest and lowest sound pressures in the room. In spaces with parallel walls, no furniture or fittings, and low sound absorption, repeated reinforcement and cancellation may lead to very strong nodes and antinodes.

The resonance frequencies of a box-shaped room can be calculated according to

$$
f_{n_{x} n_{y} n_{z}}=\frac{c_{0}}{2}\left[\left(\frac{n_{x}}{L_{x}}\right)^{2}+\left(\frac{n_{y}}{L_{y}}\right)^{2}+\left(\frac{n_{x}}{L_{z}}\right)^{2}\right]^{0.5}
$$

Where $c_{0}$ is the speed of sound $(\mathrm{m} / \mathrm{s}) ; \mathrm{L}_{\mathrm{x}}, \mathrm{L}_{\mathrm{y}} \mathrm{L}_{z}$ are the characteristic dimensions of room $(\mathrm{m})$ and $\mathrm{n}_{\mathrm{x}}, \mathrm{n}_{\mathrm{y}}, \mathrm{n}_{\mathrm{z}}$ are equal to $1,2,3, \ldots \ldots$. Eq. (1) does not give information about the spatial variation of SPL at the resonance frequencies or the location of the maximum and minimum sound pressure levels (SPLs). Sophisticated modelling methods which solve the wave equation of sound at every point in the space can be applied. Modes occurring along one dimension of the room are called axial modes. If the room walls are parallel and $\mathrm{Lx}>$ Ly $>$ Lz, the first axial mode lmn=100 occurs along the length of the room. Its node would be in the middle of the room and the antinodes by the walls. According to Eq. (1) the first resonance mode occurs at the frequency whose wavelength is 
half the length of the room. The second axial mode would be $1 \mathrm{mn}=200$ if $\mathrm{Lx}>2 \mathrm{Ly}$, otherwise $\mathrm{lmn}=010$. Modes happening along two dimensions of the room are called tangential modes, i.e. $l m n=110$ or $l m n=201$. Finally, oblique modes occur along the three dimensions, i.e. $1 \mathrm{mn}=111$ which runs along the grand diagonals from opposite corners. A representation of the axial, tangential and oblique modes is shown in figure (1).

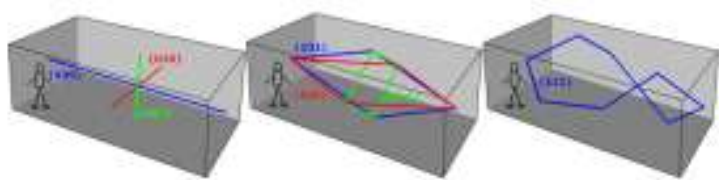

Figure 1: Left) Axial modes (100), (010) and (001). Center) Tangential modes(110), (011) and (101). Right) Oblique mode (111).

Room modes are mostly responsible of spatial SPL variations within rooms. Spatial variations are the largest at low frequencies where the lowest room modes occur. When the sound field is studied one frequency at a time, the strongest SPL differences between different points are expected at the resonance frequencies. When the measurements are performed in frequency bands, e.g. 1/3-octave bands, the variation of SPLs becomes smaller because several room modes belong to the same frequency band and they interfere with each other producing a more diffuse field. Ideally, the diffuse sound field presupposes that the sound enters to any point of the room evenly from all directions, that is, the sound intensity vector is zero. The density of room modes increases with the frequency. Therefore, the higher the frequency is, the smaller is the spatial variation of SPL, and the more diffuse becomes the sound field. The spatial variation of SPL means the difference between the maximum and minimum SPL.

\subsection{Axial room modes}

Axial modes: Involve two parallel surfaces - opposite parallel walls, or the floor and ceiling. These are the strongest modes. There are always sound pressure maxima (anti-nodes) at the walls.

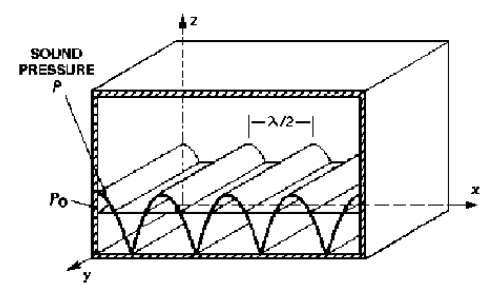

Figure 2: Represent axial mode

\subsection{Tangential room modes}

Tangential modes : Involve two sets of parallel surfaces - all four walls, or two walls the ceiling and the floor. These are about half as strong (energy) as the axial modes $(-3 \mathrm{~dB})$. There are always sound pressure maxima (anti-nodes) at the walls.

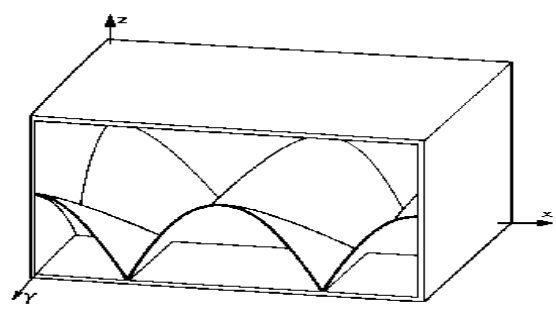

Figure 3: Represent tangential mode

\subsection{Oblique room modes}

Oblique modes : Involve all six surfaces - four walls, the ceiling and the floor. These are about one quarter as strong (energy) as the axial modes, and half as strong as the tangential modes $(-6 \mathrm{~dB})$. Oblique modes are rarely much relevant. There are always sound pressure maxima (antinodes) at the walls.

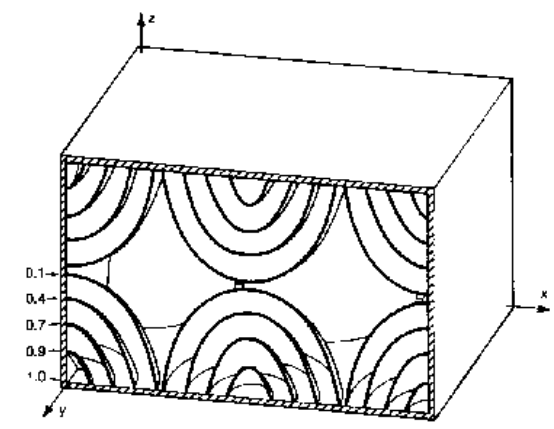

Figure 4: Represent oblique mode

\section{MATERIALS AND METHODS}

The experimental part of this paper aimed to solid understanding about the distribution of low frequency noise inside laboratory of Shopra Elkheima electrical power plant, what is the location of the maximum and minimum SPLs, what is the variation of SPL, and how different parameters, like dimensions of the room, amount of fittings and absorption, affect the spatial variation of SPL. Experimental methods and modelling methods were applied.

\subsection{Measurement of the noise:}

Sound level Meter model 2260 (Brüel \&Kjaer, Denmark) of accuracy $0.7 \mathrm{~dB}$ and resolution $0.1 \mathrm{~dB}$ is used to measure low frequency noise in different places. By analyzing the spectra of acoustic noise frequencies, the equipment was positioned at a height of $1.2 \mathrm{~m}$ above the ground.

\subsection{Model analysis}

With the help of Comsol multiphysics ${ }^{\circledR}$ software $4.3 \mathrm{~b}$.

\section{RESULTS AND DISCUSSION}

\subsection{Sound frequency analysis}

Noise analysis was performed at Laboratory near turbine in electric (Shopra Elkheima)[4]. Figure 5 shows the noise spectra measured at laboratory within frequency range of 16$250 \mathrm{~Hz}$. It was found that the difference between $\mathrm{L}_{\mathrm{C}}-\mathrm{L}_{\mathrm{A}}$ for at laboratory exceed $15 \mathrm{~dB}$. This difference is an indicator for 
International Journal of Science and Engineering Applications

Volume 4 Issue 4, 2015, ISSN-2319-7560 (Online)

the presence of the amount of low frequency noise energy in the noise. From figure 5, we can found that turbine which is near laboratory is the source of low frequency noise. It is clear also that the sound pressure level in $1 / 3$ octave band is $5 \mathrm{~dB}$ or more between some of neighboring bands, the noise is said $t$ be tonal in this case.

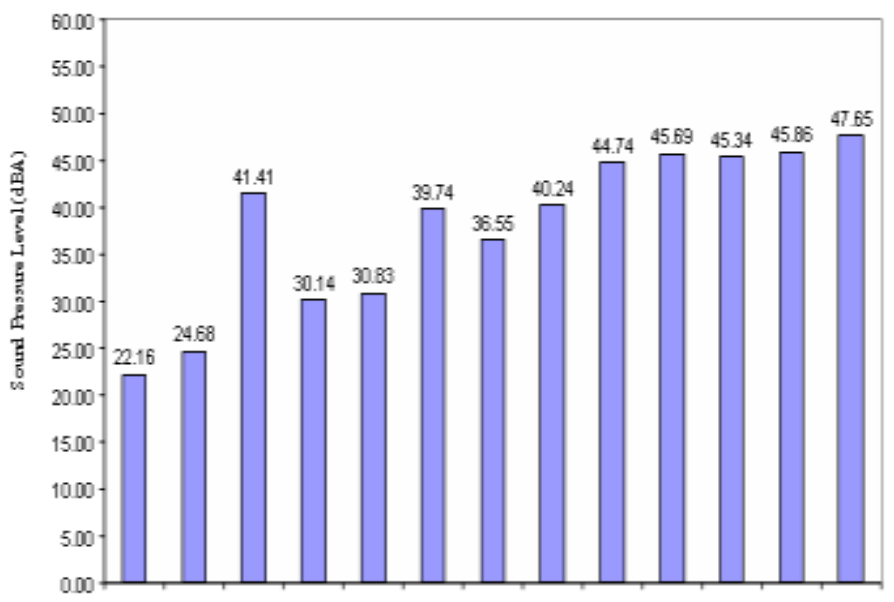

$16.00 \quad 20.00 \quad 25.00 \quad 31.50 \quad 40.0050 .00 \quad 63.00 \quad 80.00100 .00125 .0016000200 .00250 .00$ 130 dave Bend Freguency (Hz)

Figure 5: Represents the noise spectra measured at laboratory in Shopra Elkheima electrical power plant.

Table 1: Represent laboratory modes.

\begin{tabular}{|c|c|c|c|c|}
\hline \multirow{2}{*}{ Freq.(Hz) } & \multicolumn{3}{|c|}{ Mode index } & \multirow{2}{*}{ Type } \\
\hline & $\mathbf{n}_{\mathbf{x}}$ & $\mathbf{n}_{\mathbf{y}}$ & $\mathbf{n}_{\mathbf{z}}$ & \\
\hline 29.31 & 1 & 0 & 0 & \multirow{17}{*}{ Axial } \\
\hline 48.57 & 0 & 1 & 0 & \\
\hline 56.66 & 0 & 0 & 1 & \\
\hline 58.62 & 2 & 0 & 0 & \\
\hline 87.93 & 3 & 0 & 0 & \\
\hline 97.14 & 0 & 2 & 0 & \\
\hline 113.33 & 0 & 0 & 2 & \\
\hline 117.24 & 4 & 0 & 0 & \\
\hline 145.71 & 0 & 3 & 3 & \\
\hline 146.55 & 5 & 0 & 0 & \\
\hline 170.0 & 0 & 0 & 3 & \\
\hline 175.86 & 6 & 0 & 0 & \\
\hline 194.28 & 0 & 4 & 0 & \\
\hline 205.17 & 7 & 0 & 0 & \\
\hline 226.66 & 0 & 0 & 4 & \\
\hline 234.48 & 8 & 0 & 0 & \\
\hline 242.85 & 0 & 5 & 0 & \\
\hline 56.72 & 1 & 1 & 0 & \multirow{7}{*}{ Tangential } \\
\hline 63.79 & 1 & 0 & 1 & \\
\hline 74.63 & 0 & 1 & 1 & \\
\hline 76.12 & 2 & 1 & 0 & \\
\hline 81.53 & 2 & 0 & 1 & \\
\hline 101.46 & 1 & 2 & 0 & \\
\hline 112.46 & 0 & 2 & 1 & \\
\hline
\end{tabular}

\begin{tabular}{|l|l|l|l|}
\hline 117.06 & 1 & 0 & 2 \\
\hline 123.30 & 0 & 1 & 2 \\
\hline 146.53 & 1 & 3 & 0 \\
\hline 152.25 & 4 & 2 & 0 \\
\hline 172.50 & 1 & 0 & 3 \\
\hline 176.80 & 0 & 1 & 3 \\
\hline 187.02 & 4 & 3 & 0 \\
\hline 195.79 & 0 & 2 & 3 \\
\hline 202.93 & 2 & 4 & 0 \\
\hline 213.25 & 3 & 4 & 0 \\
\hline 223.90 & 0 & 3 & 3 \\
\hline 243.12 & 3 & 0 & 4 \\
\hline 250.19 & 4 & 0 & 4 \\
\hline 80.18 & 1 & 1 & 1 \\
\hline 94.90 & 2 & 1 & 1 \\
\hline 116.21 & 1 & 2 & 1 \\
\hline 126.73 & 1 & 1 & 2 \\
\hline 136.52 & 2 & 1 & 2 \\
\hline 152.11 & 1 & 2 & 2 \\
\hline 160.36 & 2 & 2 & 2 \\
\hline
\end{tabular}

\subsection{Acoustic behavior}

To illustrate the effects, we are going to calculate every eigenfrequencies below $200 \mathrm{~Hz}$, together with their corresponding eigenmodes. Table 1 shows both eigenfrequencies and eigenmodes of the laboratory.

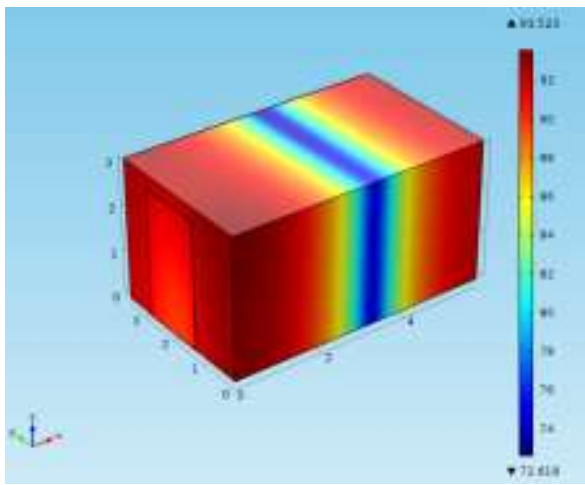

(a) Mode(1,0,0) frequency (28.3)

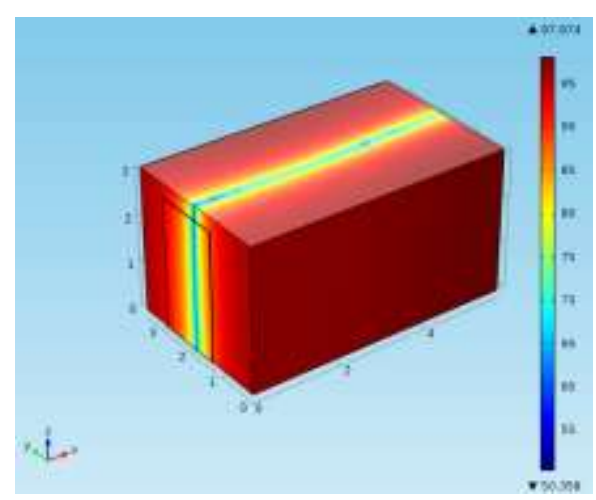

(b) Mode $(0,0,1)$ frequency (43.19) 


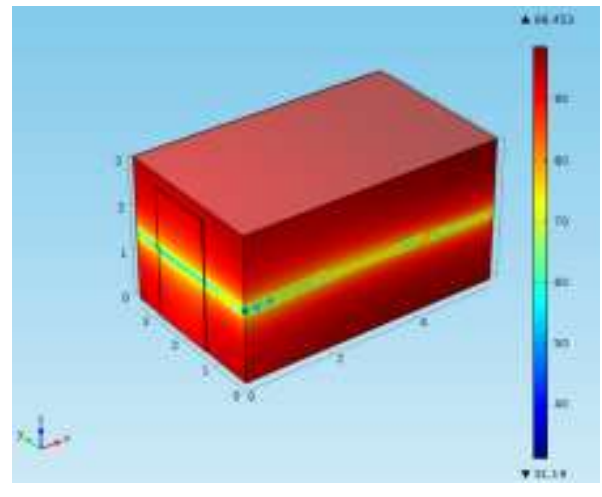

(c) $\operatorname{Mode}(0,1,0)$ frequency $(49.14)$

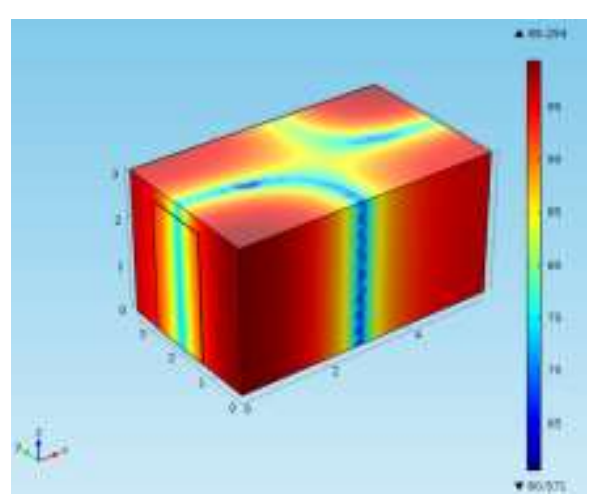

(d) Mode $(1,1,0)$ frequency $(50.66)$

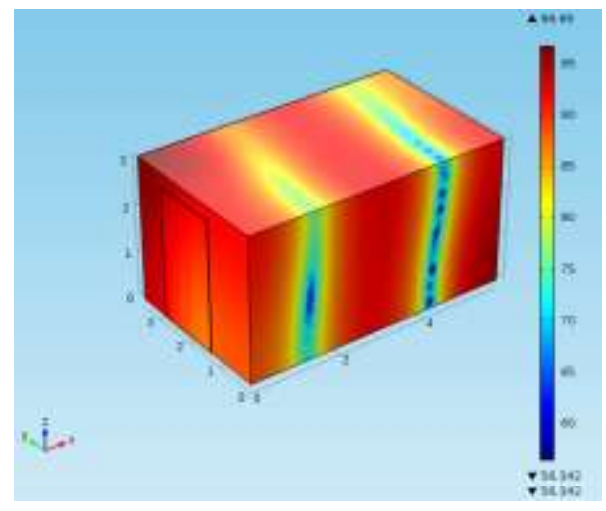

(e) $\operatorname{Mode}(2,0,0)$ frequency $(53.56)$

Figure 6: Shows a few mode shapes a,b,c,d and e.

From figure 6 we can found that if source is at a node, it cannot excite the mode; but if it is at an antinode, it gives maximum excitation of that mode. At a node, microphone reads nothing; at an antinode, it gets maximum reading for that mode. It is clear that eigenmodes differ slightly from the exact solution for an empty room. To eliminate the generation of standing wave is by building rooms with no perpendicular walls or with dimensions which are not multiples of $\lambda / 2$ of source characteristic wavelength.

\section{CONCLUSION}

This work demonstrates the capabilities of COMSOL Multiphysics to solve the complex problem of acoustic performance of rooms. Most rooms have their fundamental resonances in $20-200 \mathrm{~Hz}$ region.

\section{REFERENCES}

[1] Pawlaczyk-Luszczynska M, Dudarewicz A, Szymczak W, Sliwinska-Kowalska M. 2010. Evaluation of annoyance from low frequency noise under laboratory conditions. J. Noise Health, 12, 166-81.

[2] Ziaran S.(2011). Effects of low frequency noise in closed space on the human., In: Proceedings of the Inter-Noise 2011. The 40th International Congress and Exposition on Noise Control Engineering. Osaka: Publisher INCE/J and ASJ; 2011. p. 8, abstract p. 211.

[3] Low frequency noise 2001. Technical Research Support for DEFRA. Noise Program.

[4] A.M.Shehap, Hany A.Shawky and Tarek M.El-Basheer, 2015. A study of the whole body vibration analysis induced by low frequency noise and vibrations in Egyptian power plants. 22nd International Congress on Sound and Vibration, Florence, Italy.12-16 July. 